\title{
Ensinando a Escala de Braden como estratégia para melhoria da qualidade da assistência em enfermagem
}

\author{
Teaching the Braden Scale as a Strategy for Improving \\ the Quality of Nursing Care
}

\author{
Winnie Taise Pena Macêdo', Brena Melo Figueiredo', Danielle Saraiva Tuma dos Reis² \\ 'Faculdade de Enfermagem, Universidade Federal do Pará, Belém (PA), Brasil. \\ ${ }^{2}$ Hospital Universitário João de Barros Barreto, FAENF, Universidade Federal do Pará, Belém (PA), Brasil.
}

\begin{abstract}
RESUMO
Contextualização: As taxas de eventos adversos servem como indicadores de qualidade dos serviços de saúde. Um dos indicadores de qualidade em saúde é a ausência ou presença de Lesão por Pressão, esta causa transtornos físicos, emocionais ao paciente, caracterizando um enorme desafio para as instituições de saúde devido longo tempo de recuperação e permanência exigido pela lesão, elevando os custos de internação hospitalar. Descrição da experiência: Trata-se de um estudo descritivo, do tipo relato de experiência de atividades desenvolvidas em um Projeto de Extensão e teve como principal objetivo capacitar a equipe de enfermagem para o correto preenchimento da Escala de Braden em busca do cuidado seguro aos pacientes atendidos em uma clínica em um Hospital público de Belém-PA, no período de março/2016 a fevereiro/2017. Foram capacitados 43 profissionais de enfermagem dos turnos manhã, tarde e noite, sendo 8 enfermeiros, 16 técnicos e 19 auxiliares de enfermagem. Primeiro foi realizado análise quantitativa e qualitativa das escalas, para avaliar se as mesmas estavam sendo preenchidas corretamente. Posteriormente foi realizado o treinamento da equipe, e em seguida, uma nova análise para avaliar o impacto das capacitações. Considerações finais: Após os treinamentos, verificou-se melhora significativa no preenchimento da escala de Braden, pois o número de pacientes avaliados incorretamente reduziu 20\%, e o número de escalas não avaliadas reduziu $14 \%$. Houve uma discreta evolução do preenchimento parcial das escalas de apenas $2,6 \%$. Esses números mostram que as escalas não somente estão sendo preenchidas, bem como nos permite afirmar que a qualidade dessa avaliação também melhorou. Com isso, conclui-se que a educação permanente em saúde é de fundamental importância e precisa ser uma constante nos serviços hospitalares, para que cada vez mais os profissionais se conscientizem e se comprometam a desenvolver uma assistência de enfermagem mais segura, garantindo maior qualidade ao cuidado prestado aos pacientes.
\end{abstract}

PALAVRAS-CHAVE: Educação continuada. Úlcera por pressão. Segurança do paciente.

\section{ABSTRACT}

Background: The rates of adverse events are used as indicators of quality of healthcare services. One of the indicators of healthcare quality is the absence or presence of Pressure Ulcer, which causes physical and emotional disorders to the patient, presenting a huge challenge for health institutions due to the long recovery time and permanence required by the injury, raising the costs of the hospital stay. Description of the experience: This is a descriptive study, based on a experience report about the activities developed in an Extension Project and the main goal of this study was to enable the nursing team to correctly fill the Braden Scale, in order to ensure safe patient care to the patients treated at a clinic in a public hospital in Belém-PA. It was developed from March/2016 to February/2017. A total of 43 nursing professionals were trained in the morning, afternoon and evening shifts, with 8 nurses, 16 technicians and 19 nursing assistants. First, a quantitative and qualitative analysis of the scales was carried out to evaluate if they were being correctly filled. Subsequently the team training was carried out, and then a new analysis was made to evaluate the impact of the training. Final considerations: After the training, there was a significant improvement in the Braden score, since the number of patients evaluated incorrectly reduced by $20 \%$, and the number of non-evaluated scales reduced by $14 \%$. It was found a slightly evolution of the partial filling of the scales of only $2.6 \%$. These numbers show that scales are not only being filled, they also allow us to state that the quality of this assessment has also improved. Therefore, it was concluded that the permanent education in health is of fundamental importance and must be a constant in the hospital services, so that more and more professionals become aware and committed to develop a safer nursing care, guaranteeing higher quality of care to the patients.

KEYWORDS: Education, Continuing. Pressure ulcer. Patient safety. 


\section{Contextualização}

A qualidade da assistência no ambiente hospitalar é uma preocupação reconhecida na prática das equipes de enfermagem, que prezam por uma prestação de cuidado seguro. Nos últimos anos, tem se observado um aumento no desenvolvimento de ações em torno dessa temática, sendo assim um reflexo de políticas nacionais e internacionais, protocolos institucionais baseados em evidências científicas, fundamentais para a efetivação de uma cultura de segurança nos serviços de saúde, visando a prevenção de incidentes e danos decorrentes de falhas na assistência à saúde ${ }^{1-4}$.

Com o forte interesse em se estudar essa temática, cresce cada vez mais o volume de publicações na área. No entanto, quando os primeiros estudos surgiram, houve uma inconsistência na linguagem adotada, comprometendo a compreensão de alguns trabalhos. Diante disso, a Organização Mundial da Saúde criou uma classificação de segurança do paciente, e a partir daí, um grupo de trabalho desenvolveu a Classificação Internacional de Segurança do Paciente (International Classification for Patient Safety - ICPS), que trouxe algumas definições relacionadas a esse universo ${ }^{5}$.

O ICPS define "Erro" como uma falha em executar um plano de ação, e pode ser classificado como: não-intencional e intencional. Já o "incidente" relacionado ao cuidado de saúde é um evento que poderia ter resultado, ou resultou, em dano desnecessário ao paciente. Os incidentes classificam-se como: near miss (incidente que não atingiu o paciente); incidente sem dano; incidente com dano ou evento adverso ${ }^{6}$.

A segurança do paciente acabou por tornar-se uma das principais metas dos serviços de saúde, pois os custos decorrentes da falta de segurança e os danos ocasionados aos pacientes, geram grandes impactos no cuidado. Anualmente, cerca de 42,7 milhões de incidentes com dano ocorrem no mundo. Diante de dados tão expressivos, as organizações de saúde têm discutido cada vez mais sobre essa temática, em buscas de reduzir esse número? ${ }^{7}$.

O Brasil também tem se organizado para contribuir para a melhoria da segurança do paciente, a exemplo disso, o Ministério da Saúde por meio da Portaria $n^{\circ} 529$, instituiu em 2013, o Programa Nacional de Segurança do Paciente (PNSP) que tem como principal objetivo promover a qualidade do cuidado em saúde em todos os estabelecimentos de saúde do país ${ }^{8}$.

As taxas de ocorrência de eventos adversos servem como indicadores de qualidade dos serviços de saúde, e um dos principais indicadores em saúde é a ocorrência de Lesão por Pressão (LPP), esta constitui um importante problema para as instituições de saúde, pois o tratamento dessas feridas é muitas vezes prolongado e oneroso, além de gerar grandes repercussões na qualidade de vida dos indivíduos?.

A ocorrência de LPP está, muitas vezes, associada à qualidade da assistência prestada pela equipe de enfermagem, entretanto, trata-se de um problema multicausal e que inclui fatores extrínsecos e intrínsecos ao paciente. Assim, se faz necessário que o enfermeiro seja capaz de realizar uma avaliação criteriosa e periódica do paciente quanto ao risco de desenvolvimento de LPP. Para isso, existem vários instrumentos de avaliação de risco, reconhecidos mundialmente e já validados no Brasil, sendo a Escala de Braden a mais conhecida ${ }^{10,11}$.

O instrumento de avaliação do risco para LPP mais utilizado em todo mundo é a Escala de Braden, desenvolvida por Barbara Braden e colaboradoras em 1987², leva em consideração a fisiopatologia das lesões por pressão e possibilita a avaliação de seis parâmetros importantes no que diz respeito ao desenvolvimento deste agravo, são eles: percepção sensorial, umidade, mobilidade, atividade, nutrição, fricção e cisalhamento. Para cada item desses, é determinado uma pontuação que são somadas e resultam em um total, denominado de score ${ }^{10}$. O escore total varia de 6 a 23 pontos e a classificação pode ser: risco muito alto (escore $\leq 9)$, risco alto (10 a 12), risco moderado (13 a 14), risco baixo (15 a 18) e sem risco (19 a 23) ${ }^{12}$. 
É primordial que o enfermeiro conheça a fundo sobre essa problemática e saiba utilizar adequadamente as escalas de avaliação de risco, que são importantes instrumentos que auxiliam na tomada de decisões, além do reconhecimento de fatores de risco adicionais por meio do julgamento clínico necessário para a identificação de LPP. O conhecimento relacionado às características e a adequada avaliação das LPPs são modos de instrumentalizar a equipe de enfermagem para melhor manejo e prevenção desses agravos.

Posto a relevância do emprego da escala de Braden no ambiente hospitalar é essencial que os profissionais de enfermagem tenham o conhecimento necessário para o correto preenchimento desta escala, tendo como objetivo principal a melhoria da qualidade do cuidado prestado através da prevenção de LPP. Neste sentido, o presente estudo tem por objetivo relatar as experiências de acadêmicas de enfermagem, na execução de um projeto de extensão com a finalidade de promover educação em saúde na busca de uma assistência de enfermagem segura sob o indicador de qualidade prevenção de LPP.

\section{Descrição da experiência}

Trata-se de um estudo descritivo, do tipo relato de experiência de atividades desenvolvidas por acadêmicas de enfermagem do oitavo semestre da Universidade Federal do Pará durante a execução de um Projeto de Extensão vinculado a Pró-Reitoria de Extensão (PROEX-UFPA), intitulado: “Segurança do Paciente Portador de Doenças Transmissíveis: uma Assistência de Enfermagem de Qualidade" no qual tinha como principal objetivo avaliar o preenchimento da Escala de Braden pela equipe de enfermagem antes e após a capacitação em busca do cuidado seguro aos pacientes atendidos na clínica de Doenças Infecto-parasitárias (DIP) para prevenção de LPP. Na referida clínica, se tem disponível entre os impressos do prontuário do paciente, a Prescrição de Enfermagem, que contempla a escala de Braden.

O Projeto de Extensão foi realizado na clínica de Doenças Infecto-parasitárias em um Hospital Universitário de Belém-PA, no período de março/2016 a fevereiro/2017. O presente estudo obedeceu os preceitos éticos de pesquisa com seres humanos disciplinados pela Resolução 466/2012, e foi aprovado pelo Comitê de Ética da instituição, evidenciado através do parecer de n ${ }^{\circ} 1.911 .829$.

Foi investigado o perfil dos funcionários da clínica através de um questionário semiestruturado, que continha itens como: sexo, idade, função e por fim a seguinte pergunta: O que você acha a respeito da Escala de Braden presente no impresso da SAE?

O estudo foi constituído por três etapas: 1- Diagnóstico situacional anterior às capacitações a fim de avaliar o correto preenchimento da escala de Braden nos impressos; 2- Capacitação da equipe de enfermagem sobre a Escala de Braden; 3-Diagnóstico situacional após as capacitações para avaliar o impacto da capacitação.

O diagnóstico situacional consistiu na análise quantitativa da Escala de Braden através da busca nos prontuários, para identificar se a escala estava sendo preenchida em todos os critérios para avaliar o risco do paciente evoluir para LPP. Foi utilizado um instrumento de coleta de dados elaborado pelas integrantes do projeto de extensão, no qual continha três categorias de classificação para a Escala de Braden, se o paciente foi AVALIADO em sua totalidade, AVALIADO PARCIALMENTE e NÃO AVALIADO em relação aos parâmetros de risco para LPP. A pesquisa foi realizada antes das capacitações, levando sempre em consideração o preenchimento da escala de Braden do dia anterior.

Quanto à análise qualitativa do impresso, verificou-se o correto preenchimento pelos enfermeiros de acordo com a condição clínica dos pacientes. As integrantes do Projeto foram divididas para avaliar cada paciente, e logo após foi efetuada a 


\section{ighe}

comparação com as avaliações dos enfermeiros. Os resultados que divergissem na classificação de risco do paciente desenvolver UPP passava por uma nova avaliação, de maneira imparcial para que assim chegasse a um resultado em comum.

As capacitações acerca do preenchimento correto da escala de Braden ocorreram em 12 encontros com os profissionais de enfermagem, de acordo com a disponibilidade de cada um, no horário de trabalho. Os encontros foram realizados em uma sala anexa à clínica e tinham a duração média de 20 a 30 minutos. Foi utilizado um dado ilustrativo no qual continha em suas faces, cada parâmetro da escala e servia para avaliar o conhecimento prévio dos funcionários. Além de um álbum seriado, que continha informações e ilustrações sobre a escala e suas dimensões, além de exemplos de como e quando pontua-la corretamente.

\section{Resultados e impactos}

\section{Diagnóstico situacional anterior às capacitações}

Foram 43 profissionais de enfermagem dos turnos manhã, tarde e noite, sendo 8 enfermeiros, 16 técnicos e 19 auxiliares de enfermagem, 36 do sexo feminino e 7 do sexo masculino, com idade entre 28 e 66 anos, a média de idade da clínica encontra-se em 46,4 anos.

Com relação ao questionamento obteve-se em sua maioria, a resposta positiva acerca da Escala de Braden, onde os funcionários responderam de um modo geral, que a Escala é um importante instrumento para prevenção de lesões, recuperação e avaliação dos pacientes acamados, que possibilita elevar a qualidade da assistência prestada através de cuidados adequados, caracterizando $56 \%$ (24). Verificou-se algumas respostas em branco (8), o que configura 18,6\%. Além disso, 25,5\% (11) das pessoas relataram não saber opinar no momento.

Quanto à análise quantitativa do preenchimento dos impressos, foram analisados 107 prontuários, em relação à Escala de Braden. 72 pacientes foram AVALIADOS quanto ao risco de LPP, representando 67\%, 11 pacientes foram AVALIADOS PARCIALMENTE, ou seja, sem o preenchimento de todos os parâmetros para a Escala, representando $10 \%$ e 24 pacientes NÃO FORAM AVALIADOS, configurando $23 \%$, como podemos visualizar no Gráfico 1.

No segundo momento, para a análise qualitativa do impresso, foram avaliados 113 prontuários, destes 56 (50\%) foram preenchidos corretamente coincidindo com o mesmo risco avaliado pelas autoras e enfermeiras, segundo a Escala de Braden, 16 pacientes (14\%) não foram avaliados pelos enfermeiros, isto é, tiveram suas escalas deixadas em branco e 41 (36\%) precisaram de uma nova avaliação, pois o risco para

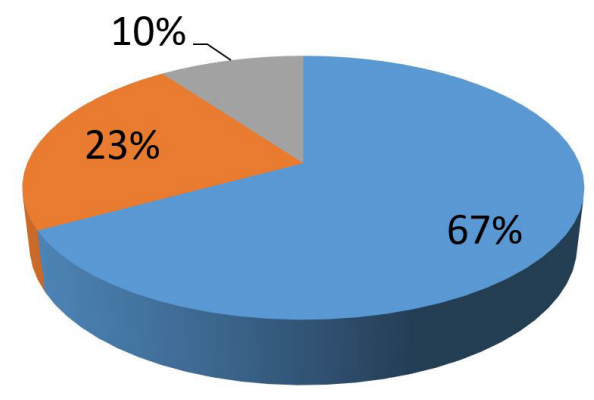

aVALIADO NÃO AVALIADO $\square$ AVALIADO PARCIALMENTE

Gráfico 1. Representação gráfica da análise quantitativa da Escala de Braden anterior às capacitações para a equipe de enfermagem, Belém-PA.

Fonte: Pesquisa das autoras. 


\section{ijhe}

LPP era discordante entre acadêmicas e enfermeiros, destas, apenas 3 estavam em concordância com a avaliação dos enfermeiros, e outras 29 concordavam com a avaliação das participantes do projeto e 1 discordou tanto da avaliação da enfermagem, quanto das extensionistas (Gráfico 2).

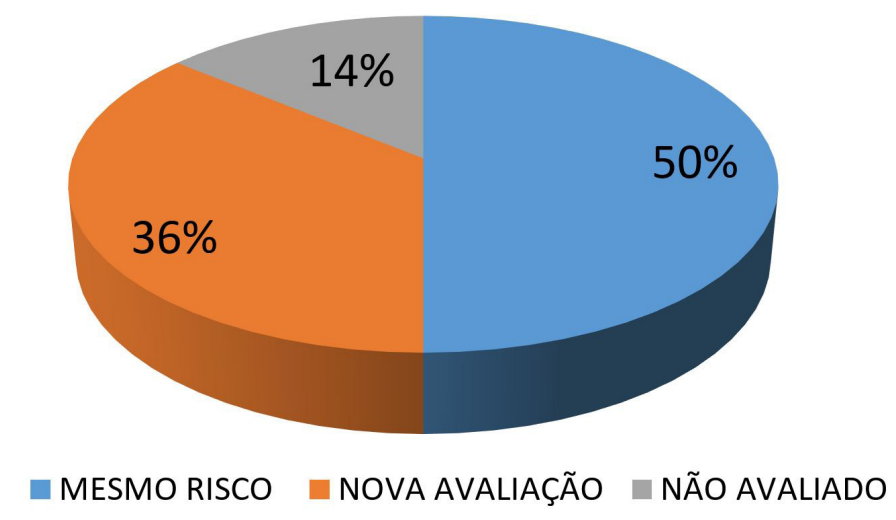

Gráfico 2. Representação gráfica da análise qualitativa da Escala de Braden anterior às capacitações para a equipe de enfermagem, Belém-PA.

Fonte: Pesquisa das autoras.

Nesta análise, podemos verificar a qualidade da avaliação realizada pelos enfermeiros desta clínica. A maioria foi dos pacientes foram avaliados corretamente segundo a escala de Braden, por meio do preenchimento adequado e fidedigno dos parâmetros. No entanto, havia um número de pacientes com a avaliação equivocada para o risco de LPP, pois o escore de risco final dado pelos enfermeiros não se encaixava com o real quadro clínico dos pacientes, deixando-os mais suscetíveis a ocorrência de lesão, ou seja, possibilidade de ocorrência de eventos adversos relacionados a assistência à saúde devido à baixa qualidade do cuidado prestado e o planejamento das ações para prevenção insuficientes.

\section{Capacitação da equipe de enfermagem sobre a Escala de Braden}

A princípio, observou-se certa apreensão por parte dos profissionais de enfermagem no momento anterior às capacitações. Pode-se perceber que uma pequena parte dos profissionais demonstrou interesse em conhecer a Escala de Braden, a maioria justificou a sobrecarga de serviço como desculpa para não participar da capacitação, além da falta de interesse por não necessitar de conhecimentos novos em razão dos anos de experiência que já possuíam. Apesar disto, foi necessária a atuação da chefia para estimular a participação de todos.

A capacitação utilizou metodologias ativas envolvendo as dimensões afetivas e intelectuais dos indivíduos, tornando o conteúdo mais próximo da realidade deles, valorizando o conhecimento individual sobre o assunto, além do uso de tecnologias educacionais como o álbum seriado ilustrado representando os conceitos para socializar o conhecimento, e figuras relacionadas com o tema em questão a fim de estimular a fixação dos conteúdos ministrados através de imagens comuns ao cotidiano deles em serviço, e um cubo ilustrativo contendo em suas faces figuras representando cada dimensão da escala de Braden a fim de verificar o conhecimento prévio dos funcionários.

Em um segundo momento, foi realizado a parte prática da capacitação com a aplicação da escala pelos funcionários. Cada profissional avaliava três pacientes com diferentes riscos para LPP com o intuito de colocar em prática o conhecimento teórico adquirido e exercitar o correto preenchimento da escala. 


\section{ijhe}

Diagnóstico situacional após as capacitações

Foi realizado uma nova análise dos prontuários para avaliar o impacto das capacitações e a adesão dos profissionais em relação ao preenchimento correto da Escala de Braden.

Quanto à avaliação quantitativa, o total de 105 prontuários analisados, 88 (84\%) foram AVALIADOS segundo a escala de Braden, ou seja, suas escalas estavam completamente preenchidas, $8(8 \%)$ pacientes foram AVALIADOS PARCIALMENTE, 9 (8\%) NÃO AVALIADOS, isto é, suas escalas estavam em branco (Gráfico 3).

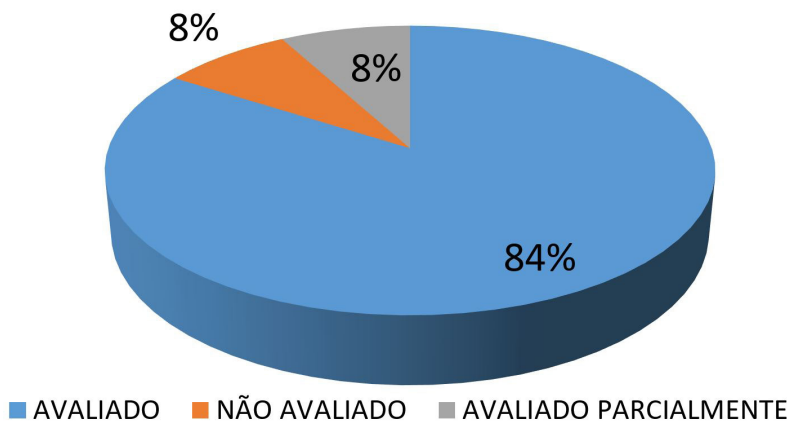

Gráfico 3. Representação gráfica da análise quantitativa da Escala de Braden posterior às capacitações para a equipe de enfermagem, Belém-PA.

Fonte: Pesquisa das autoras.

Já a análise qualitativa obteve-se os seguintes resultados: um total de 105 pacientes avaliados segundo a Escala, 73 pacientes (70\%) tiveram o mesmo risco para LPP, $11(10 \%)$ dos pacientes não haviam sido avaliados pelos enfermeiros e $21(20 \%)$ precisaram de uma nova avaliação, pois a classificação de risco não coincidia com o das pesquisadoras, como desfecho: 15 avaliações foram concordantes com as autoras, 1 avaliação concordante com a equipe de enfermagem e 4 discordaram tanto das autoras quanto da equipe de enfermagem (Gráfico 4).

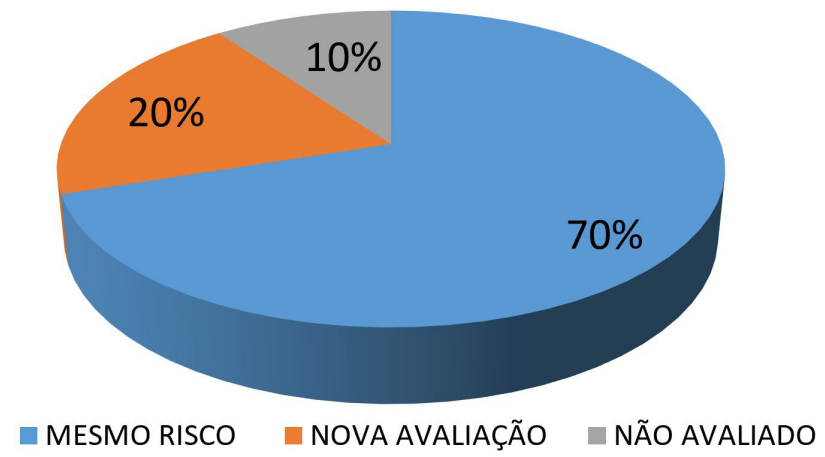

Gráfico 4. Representação gráfica da análise qualitativa da Escala de Braden posterior às capacitações para a equipe de enfermagem, Belém-PA.

Fonte: Pesquisa das autoras.

Diante dos números apresentados, conclui-se que houve melhora significativa no preenchimento do instrumento de avaliação de risco para LPP após as capacitações para a equipe de enfermagem, de $67 \%$ para $84 \%$ dos pacientes AVALIADOS pela Escala de Braden, percebeu-se também a qualidade no registro das informações com um aumento de $50 \%$ para $70 \%$ dos pacientes que apresentaram o mesmo score de 
risco segundo a avaliação das autoras em conformidade com as condições clínicas do paciente. Consequentemente reduziu o número de pacientes sem avaliação de risco para lesões, segundo os gráficos 5 e 6 .

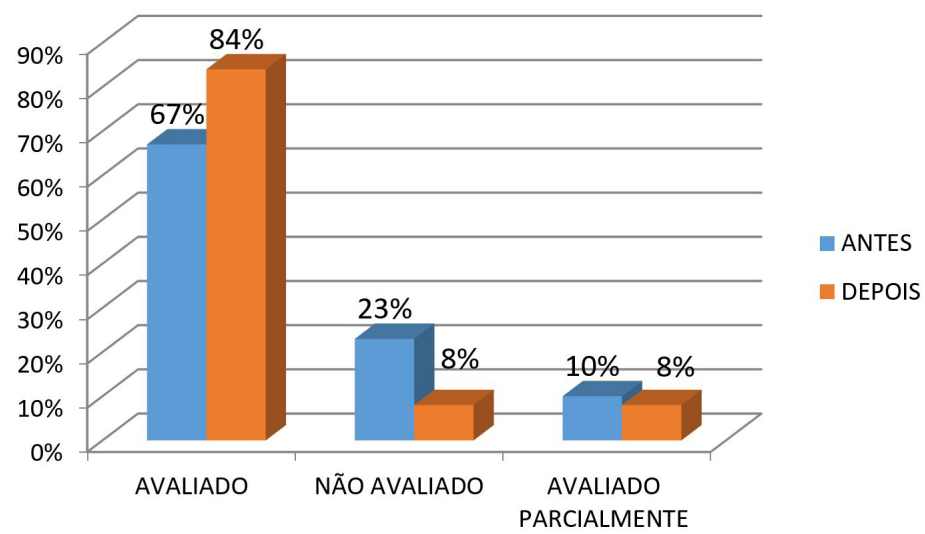

Gráfico 5. Representação gráfica da comparação quantitativa da Escala de Braden antes e após as capacitações para a equipe de enfermagem, Belém-PA. Fonte: Pesquisa das autoras.

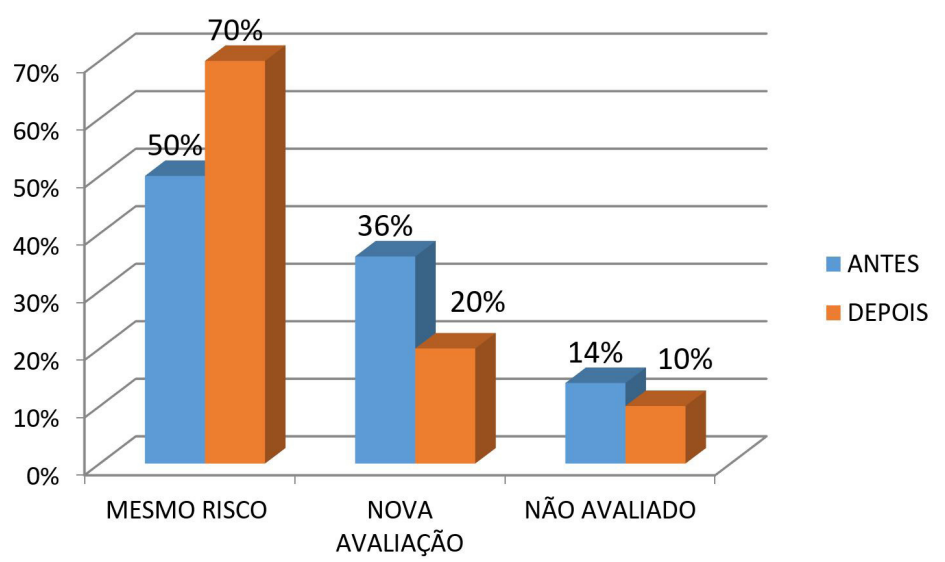

Gráfico 6. Representação gráfica da comparação qualitativa da Escala de Braden antes e após as capacitações para a equipe de enfermagem, Belém-PA.

Fonte: Pesquisa das autoras.

\section{Considerações finais}

Percebeu-se o impacto da capacitação da equipe de enfermagem em relação à aplicação da Escala de Braden, tanto quantitativamente como qualitativamente, isto configura uma melhora na prestação da assistência, com redução de eventos adversos por lesão por pressão e um aumento na indicação de medidas de prevenção.

A Educação Permanente deve ser uma proposta centrada no enfrentamento dos problemas que emergem do trabalho cotidiano nos serviços de saúde, onde o aprender e o ensinar se incorporam ao cotidiano das organizações e ao trabalho, o desafio é estimular o desenvolvimento da consciência nos profissionais sobre o seu contexto, pela sua responsabilidade em seu processo permanente de educação.

Observaram-se profissionais de enfermagem carentes de reflexão em seus locais de trabalho e principalmente com dificuldades ou até mesmo resistência em mudar padrões cuidadores já instituídos. É necessário essa mudança de postura e um comprometimento para a prestação de uma assistência de enfermagem mais segura, garantindo maior qualidade ao cuidado prestado aos pacientes.

A busca da qualidade na prestação de serviços à saúde é uma necessidade técnica e social. É preciso instrumentalizar a organização de saúde de tal forma que seja 
assegurado ao paciente que toda organização estará voltada para maximizar os cuidados e benefícios e minimizar os riscos.

A experiência vivenciada pelas autoras foi bastante desafiadora, tanto pela recepção da equipe de enfermagem quanto pela adesão às novas medidas de prevenção. $O$ profissional enfermeiro deve se empoderar dessa função formadora e de liderança de sua equipe com vistas à uma prestação de qualidade. Sem dúvida essa experiência foi significativa na formação das autoras enquanto acadêmicas e futuras enfermeiras.

Além disso, o presente estudo possuiu limitações no que tange ao número reduzido de participantes pesquisados e por se tratar de profissionais de uma única clínica, podendo não abranger a realidade de outros serviços de saúde que tenham outro perfil clínico de pacientes e cuidado, portanto, não possibilita a generalização dos resultados encontrados. Apesar disto, os resultados da pesquisa mostram-se adequados, considerando o limitado número de estudos sobre a adesão dos profissionais de enfermagem às atividades de educação em serviço.

\section{Referências}

1. Cedraz RO, Gallasch CH, Júnior EFP, Gomes HR, Rocha RG, Mininel AV. Gerenciamento de riscos em ambiente hospitalar: incidência e fatores de riscos associados à queda e lesão por pressão em unidade clínica. Esc Anna Nery [Internet]. 2018 [citado em 2018 Dez 10];22(1):1-7. Disponível em: http://www.scielo.br/pdf/ean/v22n1/pt_1414-8145-ean-2177-9465EAN-2017-0252.pdf.

2. Conselho Regional de Enfermagem (COREN). Rede Brasileira de Enfermagem e Segurança do Paciente (REBRAENSP). 10 passos para a segurança do paciente. São Paulo (SP): COREN-SP; 2010.

3. Vargas MAO, Luz AMH. Práticas seguras do/no cuidado de enfermagem no contexto hospitalar: é preciso pensar sobre isso e aquilo. Enferm Foco [Internet]. 2010; [citado em 2018 Dez 10];1(1):23-7. Disponível em: http://revista.portalcofen.gov. br/index.php/enfermagem/article/view/5

4. Tronchin DMR, Melleiro MM, Kurcgant P, Garcia AN, Garzin ACA. Subsídios teóricos para a construção e implantação de indicadores de qualidade em saúde. Rev Gaúcha Enferm [Internet]. 2009; [citado em 2018 Dez 10]; 30(3):542-6. Disponível em: http://seer.ufrgs.br/RevistaGauchadeEnfermagem/article/view/10412

5. Brasil. Agência Nacional de Vigilância Sanitária. Assistência segura: uma reflexão teórica aplicada à prática. 1. ed. Brasília: ANVISA; 2013.

6. Centro Colaborador para a Qualidade do Cuidado e Segurança do Paciente (PROQUALIS). Instituto de Comunicação e Informação Científica e Tecnológica em Saúde (ICICT/Fiocruz) [Internet]; 2012 [citado em 2018 Dez 10]. Disponível em: http://proqualis.net/.

7. Françolin L, Gabriel CS, Bernardes A, Silva AEBC, Brito MFP, Machado JP. Gerenciamento da segurança do paciente sob a ótica dos enfermeiros. Rev Esc Enferm USP [Internet]. 2015 [citado em 2018 Dez 08];49(2):277-83. Disponível em: http:// www.scielo.br/pdf/reeusp/v49n2/pt_0080-6234-reeusp-49-02-0277.pdf.

8. Brasil. Ministério da Saúde. Portaria n. 529, de $1^{\circ}$ de abril de 2013. Institui o Programa Nacional de Segurança do Paciente (PNSP). Diário Oficial da União, Brasília, DF, 2 abr. 2013.

9. Penido MF, Velasquez-Melendez G, Daros TS, Dias MA, Latini GFS. Factors associated with the incidence of pressure ulcer during hospital stay. Rev Esc Enferm USP [Internet]. 2017 [citado em 2018 Dez 03];51. Disponível em: http://www. scielo.br/scielo.php?script=sci_arttext\&pid=S0080-62342017000100421\&lng=en

10. Zambonato BP, Assis MCS, Beghetto MG. Associação das sub-escalas de Braden como risco do desenvolvimento de úlcera por pressão. Rev Gaucha Enferm. [Internet]. 2013 [citado em 2018 Dez 03];34(1):21-8. Disponível em: http://www.scielo. br/scielo.php?script=sci_arttext\&pid=S1983-14472013000200003

11. Coleman S, Gorecki C, Nelson EA, et al. Patient risk factors for pressure ulcer development: Systematic review. Int J Nurs Stud [Internet]. 2013 Jul [citado em 2018 Dez 03];50(7):974-1003. Disponível em: https://www.ncbi.nlm.nih.gov/ pubmed/23375662.

12. Borghardt AT, Prado TN, Bicudo SDS, Castro DS, Bringuente MEO. Pressure ulcers in critically ill patients: incidence and associated factors. Rev Bras Enferm [Internet]. 2016 [citado em 2019 Dez 03];69(3):431-8. Disponível em: http://dx.doi. org/10.1590/0034-7167.2016690307i.

\section{Contribuição dos autores}

Brena Melo Figueiredo participou da concepção e desenvolvimento, da elaboração do desenho metodológico, da coleta e tratamento dos dados e da redação do manuscrito. Winnie Taíse Pena Macêdo participou da concepção e desenvolvimento, da supervisão, da Análise / interpretação dos dados e da redação do manuscrito. Danielle Saraiva Tuma dos Reis participou da concepção e desenvolvimento, da supervisão, da Análise e interpretação dos dados e da redação e revisão do manuscrito. 